Available online on 15.08.2019 at http://ajprd.com
(C) 2013-19, publisher and licensee AJPRD, This is an Open Access article which permits unrestricted non-
commercial use, provided the original work is properly cited

Open $\overbrace{\text { Access }}$

Research Article

\title{
Exploring the Interaction of Tartrazine and Lipase: A Multispectroscopic Analysis, Docking and Computational Simulation Methods
}

\section{Liu Baosheng *, Zhang Hongcai, Cheng Xu}

College of Chemistry \& Environmental Science, Key Laboratory of Analytical Science and Technology, Hebei University, Baoding, PR China

\begin{abstract}
A B S T R A C T
The interaction between food colorant tartrazine and lipase was studied by multi-spectroscopic and molecular docking simulation under simulated physiological conditions to evaluate the toxic of tartrazine at the protein level. The results showed that tartrazine could effectively quench the endogenous fluorescence of lipase. The thermodynamic parameters were obtained from the van't Hoff equation, and the Gibbs free energy $\Delta G<0$, indicating that the reaction was spontaneous; $\Delta H<0, \Delta S>0$, indicating hydrophobic interaction played a major role in forming the tartrazine-lipase complex. as shown by the synchronous fluorescence, UV-vis absorption and circular dichroism data, tartrazine could lead to the conformational and micro environmental changes of lipase, which may affect its physiological function. Molecular docking results showed that tartrazine was site in the active center of lipase, which altered the microenvironment of amino acid residues at the catalytic active center of lipase.
\end{abstract}

Keywords: Tartrazine, Spectroscopic, Molecular, Docking;

A R T I C L E I N F 0: Received 01 June 2019; Review Completed 09 July 2019; Accepted 22 July 2019; Available online 15 August 2019

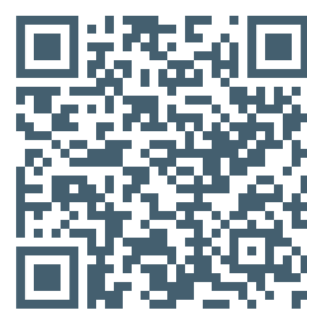

Cite this article as:

Liu Baosheng , Zhang Hongcai, Cheng Xu, Exploring the interaction of tartrazine and lipase: A multispectroscopic analysis, docking and computational simulation methods,, Asian Journal of Pharmaceutical Research and Development. 2019; 7(4):01-07, DOI: http://dx.doi.org/10.22270/ajprd.v7i4.546

*Address for Correspondence:

Baosheng Liu, College of Chemistry \& Environmental Science, Key Laboratory of Analytical Science and Technology, Hebei University, Baoding, PR China, Email: lbs @ hbu.edu.cn

\section{INTRODUCTION}

$\mathrm{T}$ lartrazine (structure formula see Fig. 1) is a synthetic acid azo dye; its aqueous solution is yellow. As a colorant, it is widely used in sugar coating and capsule of pastry, candy, beverage and pharmaceutical preparation ${ }^{1}$. Generally speaking, synthetic dyes contain azo functional groups and aromatic ring structures, which are harmful to human health. Long-term intake or intake of foods containing large amounts of tartrazine can cause allergies, migraines, eczema, anxiety, and digestive system ${ }^{2}$. Excessive intake of tartrazine may exceed the affordability of the liver and accumulate in the body, resulting in kidney and liver damage ${ }^{3}$. Some reports also suggest that tartrazine can cause allergic reactions, hyperactivity, and restlessness and sleep disorders in children ${ }^{4}$.

Lipase is a kind of hydrolytic enzyme that decomposes triacylglycerol. It belongs to intracellular enzyme. Its catalytic site contains nucleophilic catalytic triad (SHD or SHE), catalytic site is buried in the molecule. The surface is covered by a spiral cover structure formed by relatively hydrophobic amino acid residues; it has a protective effect on the catalytic site of triad ${ }^{5}$. Human lipase is mainly secreted by pancreatic acinar cells, so it is also called pancreatic lipase and plays an important role in digesting fat 
in the duodenum and in the absorption of triglycerides in the small intestine. Hydrolyzed triglycerides are converted into glycerol and fatty acids and are absorbed by human body ${ }^{6}$, which plays an important role in the process of human digestion and absorption. Zhao Lining et al. ${ }^{7}$ studied the interaction between three mercaptopropionic acidterminated CdTe quantum dots and lipase, and proved that three mercaptopropionic acid-terminated CdTe quantum dots have toxic and side effects on lipase. Zhang Rui et al. ${ }^{8}$ studied the interaction between bisphenol A and lipase in vitro in order to better understand the toxicity mechanism of bisphenol A.

In recent years, fluorescence spectroscopy has become an important means to study the mechanism of ligand-protein system, but so far, the study of lipase binding to tartrazine by a variety of spectroscopy and molecular docking techniques has not been reported. In this paper, the binding constant, binding site number and thermodynamic parameters of tartrazine-lipase system were obtained by fluorescence spectroscopy and molecular docking method. Thus, the binding mechanism of tartrazine and lipase was revealed, which provided a theoretical basis for understanding the toxicity of tartrazine to human body at the molecular level.

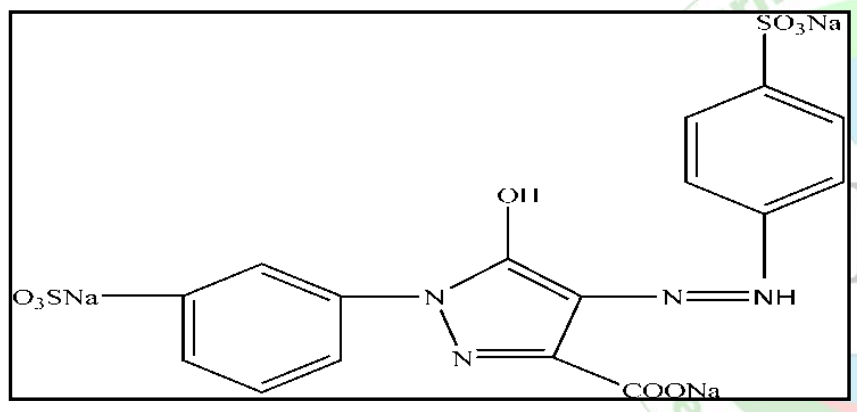

Figure 1: The structure formula of tartrazine

\section{Experimental}

\section{Apparatus}

These fluorescence spectra were acquired with a Shimadzu RF-5301PC spectrofluorophotometer. Circular dichroism spectra were recorded on a MOS-450/SFM300 circular dichroism spectrometer (Bio-Logic, France). Absorption was measured with an UV-vis recording spectrophotometer (UV-3600, Shimadzu, Japan). All pH measurements were made with a pHS-3C precision acidity meter (Leici, Shanghai, China). All temperatures were controlled by a SYC-15 B superheated water bath (Sangli, Nanjing, China).

\section{Materials}

Porcine pancreatic lipase (PPL) was purchased from SigmaAldrich (purity grade inferior 99\%, Shanghai, China), standard solution $\left(5.0 \times 10^{-6} \mathrm{~mol} / \mathrm{L}\right)$ was prepared. Tartrazine (TTZ) standard solution $\left(1.0 \times 10^{-3} \mathrm{~mol} / \mathrm{L}\right)$ was prepared. Tris-HCl buffer solution was used to keep the $\mathrm{pH}$ of the solution at 7.40 , containing $\mathrm{NaCl}(0.15 \mathrm{~mol} / \mathrm{L})$ to maintain the ionic strength of the solution. All other reagents were analytical grade and all aqueous solutions were prepared with newly double-distilled water and stored at $277 \mathrm{~K}$.
The fluorescence intensity measured in the experiment was corrected by the "internal filter effect" Eq.(1) ${ }^{9}$ :

$$
I_{c o r}=I_{o b s} \times e^{\left(A_{e x}+A_{e m}\right) / 2}
$$

Where $I_{\text {cor }}$ and $I_{\mathrm{obs}}$ are the fluorescence intensities corrected and observed, respectively, and Aex and Aem are the absorption of the system at the excitation and the emission wavelength, respectively. The intensity of fluorescence used in this paper is the corrected fluorescence intensity.

\section{Results and discussion}

\section{Experiment procedure}

\section{Fluorescence measurements}

$1.0 \mathrm{~mL}$ Tris- $\mathrm{HCl}$ buffer solution, $2.0 \mathrm{~mL}$ PPL $\left(5.0 \times 10^{-6}\right.$ $\mathrm{mol} / \mathrm{L}$ ), and different volume of TTZ were added into 10.0 $\mathrm{mL}$ colorimetric tube successively. The samples were diluted to scaled volume with double-distilled water, mixed thoroughly by shaking, and kept static for $30 \mathrm{~min}$ at different temperatures $(298 \mathrm{~K}, 310 \mathrm{~K}$, and $318 \mathrm{~K})$. Excitation wavelength with excitation and emission slit at 5 $\mathrm{nm}$ for PPL was $280 \mathrm{~nm}$ and $295 \mathrm{~nm}$, respectively. The fluorescence spectra were measured (emission wavelengths of $280-450 \mathrm{~nm}$ ). At the same time fixed $\Delta \lambda=15 \mathrm{~nm}$ or $\Delta \lambda=60 \mathrm{~nm}$, the synchronous fluorescence spectra of PPL and TTZ were recorded.

\section{Circular dichroism measurements}

A total of $1.0 \mathrm{~mL}$ Tris- $\mathrm{HCl}$ buffer solution, $2.0 \mathrm{~mL}$ PPL solution $\left(5.0 \times 10^{-6} \mathrm{~mol} / \mathrm{L}\right)$, and different volumes of TTZ were added into a $10.0 \mathrm{~mL}$ colorimetric tube successively. The samples were diluted to scaled volume with water, mixed thoroughly by shaking, and kept static for $30 \mathrm{~min}$ at 298 K. Each spectrum was recorded at wavelengths between 190 and $300 \mathrm{~nm}$ and a scan speed of $1 \mathrm{~nm} / \mathrm{s}$.

\section{UV-vis measurements}

$1.0 \mathrm{~mL}$ Tris-HCl buffer solution, $2.0 \mathrm{~mL}$ PPL solution $\left(5.0 \times 10^{-6} \mathrm{~mol} / \mathrm{L}\right)$ and different volume of TTZ were successively added to a $10.0 \mathrm{~mL}$ colorimetric tube, and the reference solutions were the corresponding concentration of TTZ solutions. The samples were diluted to scaled volume with water, mixed thoroughly by shaking, and kept static for $30 \mathrm{~min}$ at $298 \mathrm{~K}$. The UV-vis absorption spectrum of TTZ in the presence and absence of PPL were scanned with $1 \mathrm{~cm}$ quartz cells over the range from $190 \mathrm{~nm}$ to $400 \mathrm{~nm}$.

\section{Molecular docking}

The two-dimensional structure of TTZ was drawn in ChemDraw 15.0, and its three-dimensional structure was optimized by model of molecular mechanics in ChemDraw 3D. The crystal structure of PPL used for molecular docking was obtained from protein data bank (PDB ID: 1OIL). Waters and all other HETATM molecules were removed from the PDB file of PPL. Polar hydrogen atoms and Gasteiger charges were added to prepare the PPL molecule for docking. Protein-ligand docking was performed with the rigid docking tool in the Autodock 4.2.6. In this work, the selection of flexible residues for the induced fit is based on the active site of the PPL. The most 
favorable docking model was selected according to the binding energy and the geometry matching ${ }^{10}$.

\section{Results and Discussion}

The Fluorescence spectra of TTZ-PPL system

At $280 \mathrm{~nm}$ wavelength, the Trp and Tyr residues in PPL are excited, whereas a wavelength of $295 \mathrm{~nm}$ excites only $\operatorname{Trp}$ residues ${ }^{11}$. Fig. 2 showed that the fluorescence emission spectrum of PPL when TTZ was added at different concentrations. A typical emission peak was observed at about $348 \mathrm{~nm}$ when the excitation wavelength was $\lambda_{e x}=280$ $\mathrm{nm}$. (The fluorescence emission spectrum of TTZ-PPL was similar to $\lambda_{e x}=295 \mathrm{~nm}$, but the fluorescence intensity was low.) As the TTZ concentration increased, the degree of fluorescence quenching increased, and the maximum emission peak was blue-shifted, indicating that TTZ interacted with PPL and formed a stable complex ${ }^{12}$.

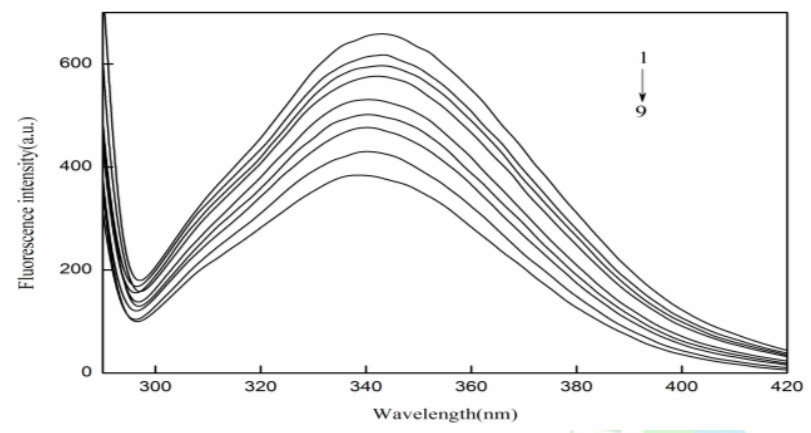

Figure 2: Fluorescence emission spectra of TTZ-PPL system ( $T=310 \mathrm{~K}$, $\left.\lambda_{e x}=280 \mathrm{~nm}\right)$

$C_{\mathrm{PPL}}=1.0 \times 10^{-6} \mathrm{~mol} / \mathrm{L}, 1 \sim 9 C_{\mathrm{TTZ}}=(0,0.1,0.5,1.5,2.0,2.5,3.0,3.5,4.0) \times 10^{-5}$ $\mathrm{mol} / \mathrm{L}$

In order to study the quenching mechanism, the quenching data was processed according to the Stern-Volmer Eq. (2) ${ }^{13}$, and the results were shown in Table 1.

$I_{0} / I=1+k_{q} \tau_{0}[L]=1+K_{s v}[L]=1+K_{D}[L]$

Where, $I_{0}$ and $I$ are the fluorescence intensities of PPL in the absence and presence of the TTZ, respectively. $k_{q}$ is the bimolecular quenching constant and $[L]$ is the concentration of the quencher, $\tau_{0}$ is the average lifetime of fluorescence (about $10^{-8} \mathrm{~s}$ ) and $K_{s v}$ is the Stern-Volmer quenching constant. From Table 1 , it could be seen that the $k_{q}$ value was greater than $2.0 \times 10^{10} \mathrm{~L} / \mathrm{mol} \cdot \mathrm{s}$ at different temperatures ${ }^{14}$.The $K_{s v}$ value was inversely correlated with temperatures. The result indicated that the combination process of TTZ-PPL system was a static quenching process. Stern-Volmer plots of the fluorescence of PPL quenched by TTZ at different temperatures were shown in Fig. 3. The good linear relationship reflected the existence of a single annihilation.

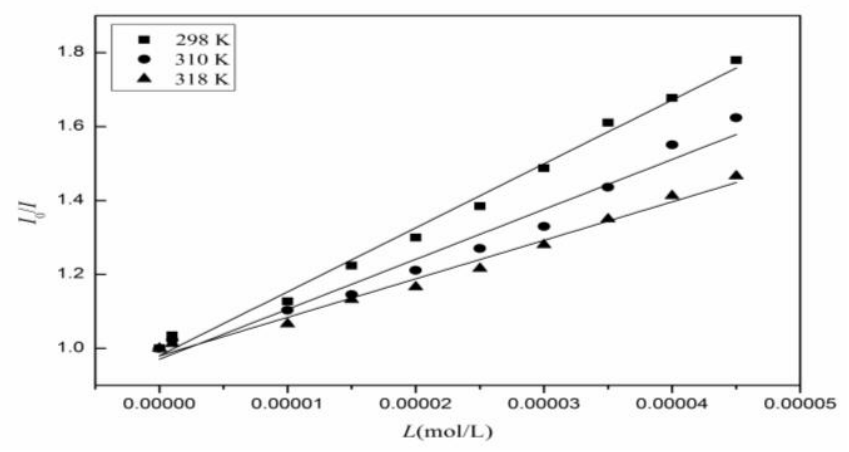

Figure 3: Stern-Volmer plots for the quenching of PPL by TTZ at different temperatures

$C_{\mathrm{PPL}}=1.0 \times 10^{-6} \mathrm{~mol} / \mathrm{L}, C_{\mathrm{TTZ}}=(0,0.1,0.5,1.5,2.0,2.5,3.0,3.5,4.0,4.5)$ $\mathrm{mol} / \mathrm{L}$

The relationship between fluorescence intensity and the concentration of quencher can usually be described using Eq. (3) ${ }^{15}$ to obtain the binding constant $\left(K_{a}\right)$ and the number of binging sites $(n)$ :

$$
\lg \left(\frac{I_{0}-I}{I}\right)=n \lg K_{a}+\mathrm{n} \lg \left\{[L]-n \frac{I_{0}-I}{I_{0}}\left[B_{t}\right]\right\}
$$

Where $[L]$ and $\left[B_{t}\right]$ are the concentrations of TTZ and PPL, respectively. On the assumption that $n$ in the bracket is equal to 1 , the curve of $\lg \left(I_{0^{-}} I\right) / F$ versus $\lg \left\{[L]-n\left[B_{t}\right]\left(I_{0^{-}} I\right) / I\right\}$ is drawn and linearly fitted, then the value of $n$ can be obtained from the slope of the plot. On the basis of value of $n$ obtained, the binding constant $K_{a}$ can also be determined. The results were listed in the Table 1 . The results showed that all the values of $n$ were approximately equal to 1 at different temperatures, indicating that TTZ had only one high-affinity binding site when bound to PPL ${ }^{16}$, that was, TTZ and PPL formed a 1:1 complex. When the temperature rose, the binding constant $K_{a}$ value was in the order of $10^{4}$ and showed a decreasing trend, and the binding ability of TTZ to PPL was reduced, which further proved that the TTZ-PPL system was static quenching.

Table 1: Quenching reactive parameters of TTZ-PPL system at different temperatures

\begin{tabular}{|l|l|l|l|l|l|l|l|}
\hline$\lambda_{e x}(\mathrm{~nm})$ & $T /(\mathrm{K})$ & $K_{s v}(\mathrm{~L} / \mathrm{mol} \cdot \mathrm{s})$ & $k_{q}(\mathrm{~L} / \mathrm{mol})$ & $r_{I}$ & $K_{a}(\mathrm{~L} / \mathrm{mol})$ & $n$ & $r_{2}$ \\
\hline$\lambda_{e x=} 280$ & 298 & $1.93 \times 10^{4}$ & $1.86 \times 10^{12}$ & 0.9953 & $2.11 \times 10^{4}$ & 1.21 & 0.9966 \\
\cline { 2 - 8 } & 310 & $1.75 \times 10^{4}$ & $1.61 \times 10^{12}$ & 0.9949 & $1.89 \times 10^{4}$ & 1.11 & 0.9937 \\
\cline { 2 - 8 } & 318 & $1.46 \times 10^{4}$ & $1.34 \times 10^{12}$ & 0.9978 & $1.51 \times 10^{4}$ & 0.94 & 0.9962 \\
\hline$\lambda_{e x=295}$ & 298 & $1.36 \times 10^{4}$ & $1.51 \times 10^{12}$ & 0.9912 & $1.38 \times 10^{4}$ & 1.16 & 0.9955 \\
\cline { 2 - 8 } & 310 & $1.19 \times 10^{4}$ & $1.27 \times 10^{12}$ & 0.9949 & $1.26 \times 10^{4}$ & 1.12 & 0.9948 \\
\cline { 2 - 8 } & 318 & $1.11 \times 10^{4}$ & $0.99 \times 10^{12}$ & 0.9922 & $1.08 \times 10^{4}$ & 0.89 & 0.9931 \\
\hline
\end{tabular}




\section{Type of interaction force of TTZ-PPL system}

Thermodynamic parameters of TTZ-PPL system can be calculated by the van't Hoff equations ${ }^{17}$.

$$
\begin{aligned}
& R \ln K=\Delta S-\Delta H / T \\
& \Delta G=\Delta H-T \Delta S
\end{aligned}
$$

Table 2: The thermodynamic parameters of TTZ-PPL at different temperatures

\begin{tabular}{|l|l|l|l|l|l|}
\hline system & $T /(\mathrm{K})$ & $K_{a} /(\mathrm{L} / \mathrm{mol})$ & $\Delta H /(\mathrm{kJ} / \mathrm{mol})$ & $\Delta S /(\mathrm{J} / \mathrm{mol} \cdot \mathrm{K})$ & $\Delta G /(\mathrm{kJ} / \mathrm{mol})$ \\
\hline$\lambda_{e x}=280 \mathrm{~nm}$ & 298 & $2.11 \times 10^{4}$ & \multirow{2}{*}{-12.71} & 40.15 & -24.66 \\
\cline { 2 - 3 } \cline { 5 - 6 } & 310 & $1.89 \times 10^{4}$ & & 40.75 & -25.33 \\
\cline { 2 - 3 } \cline { 5 - 6 } & 318 & $1.51 \times 10^{4}$ & & 40.05 & -25.45 \\
\hline
\end{tabular}

$R$ is the gas constant $(R=8.314 \mathrm{~J} / \mathrm{mol} \cdot \mathrm{K})$. Based on the linear fit plot of $R \ln K_{a}$ versus $1 / T$, the $\Delta H$ and $\Delta S$ values can be obtained. The results obtained are listed in Table 2 . It can be seen from Table 2 that $\Delta G<0$, indicating that the quenching reaction of TTZ to PPL was spontaneous; $\Delta S>0$, suggesting that there was hydrophobic interaction between TTZ and PPL; $\Delta H<0$ cannot be used as a sign of inter molecular electrostatic attraction, Ross pointed out that when $\Delta H \approx 0, \Delta S>0$, it could be considered as electrostatic attraction, and $\Delta H<0$ is considered as the result of hydrogen bonding ${ }^{18-20}$. Therefore, the force between TTZ and PPL was mainly hydrophobic interaction and hydrogen bonding.

\section{Synchronous fluorescence studies of TTZ-PPL system}

For synchronous fluorescence spectra of proteins, when the $\Delta \lambda$ value between the excitation and emission wavelengths

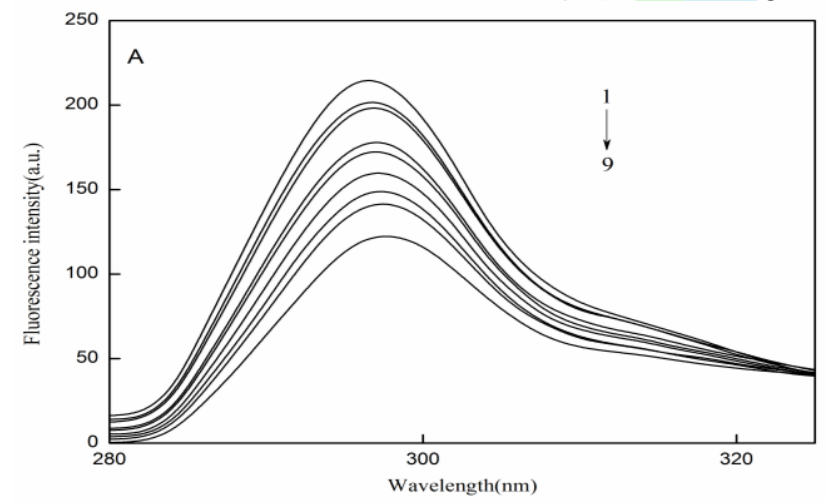

is stabilized at 15 or $60 \mathrm{~nm}$, the synchronous fluorescence gives characteristic information for Tyr or Trp residues ${ }^{21}$. Fig. 4 (A and B) showed the effects of TTZ on the synchronous fluorescence spectra of PPL. It can be seen from Fig. 4 that when $\Delta \lambda=15 \mathrm{~nm}$ or $\Delta \lambda=60 \mathrm{~nm}$, the fluorescence intensity of PPL decreased with the increase of TTZ concentration, showing that Tyr and Trp residues both participated in the interaction of TTZ with PPL. In addition, the maximum emission wavelength of Tyr and Trp residues had a slightly red shift, suggesting that the microenvironment of these residues became more hydrophobic and polarity enhancement. The results indicated that the TTZ caused a change in the conformation of the PPL.

Figure 4: synchronous fluorescence spectra of TTZ-PPL system ( $T=298 \mathrm{~K}$ ). (A) $\Delta \lambda=15 \mathrm{~nm}$; (B) $\Delta \lambda=60 \mathrm{~nm}$

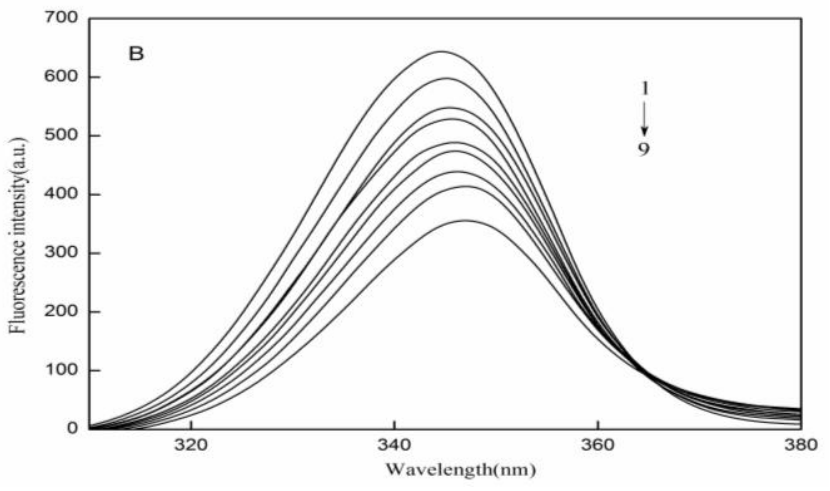
$C_{\mathrm{PPL}}=1.0 \times 10^{-6} \mathrm{~mol} / \mathrm{L}, 1 \sim 9 C_{\mathrm{TTZ}}=(0,0.1,0.5,1.5,2.0,2.5,3.0,3.5,4.0) \times 10^{-5} \mathrm{~mol} / \mathrm{L}$

UV-vis absorption spectra studies of TTZ-PPL system

The UV-vis absorption spectra of TTZ-PPL system was shown in Fig. 5. PPL had two absorption peaks, the strong absorption peak at about $208 \mathrm{~nm}$ reflects the framework conformation of the protein, the weak absorption peak at about $280 \mathrm{~nm}$ appears to be due to the aromatic amino acids (Trp, Tyr, and Phe) ${ }^{22}$. With gradual addition of TTZ to PPL solution, the intensity peak of PPL at $208 \mathrm{~nm}$ decreased with a red shift and the intensity of the peak at $280 \mathrm{~nm}$ had minimal changes (Fig. 5). The intensity of the absorption peak at $280 \mathrm{~nm}$ was reduced, illustrating that the interaction between TTZ and PPL happened in the ground-state molecules. The results showed that type of fluorescence quenching of TTZ-PPL system was static quenching ${ }^{23}$. This conclusion was consistent with the conclusion of the fluorescence quenching experiment.

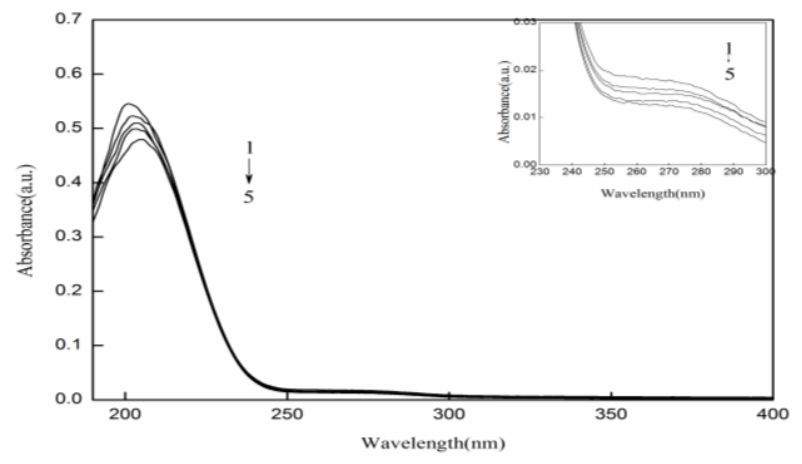

Figure 5: Absorption spectrum of TTZ-PPL system $(T=298 \mathrm{~K})$ $C_{\mathrm{PPL}}=1.0 \times 10^{-6} \mathrm{~mol} / \mathrm{L}, 1 \sim 6: C_{\mathrm{TTZ}}=(0,0.1,1.0,1.5,2.0) \times 10^{-5} \mathrm{~mol} / \mathrm{L}$ 


\section{Circular dichroism spectra studies of TTZ-PPL system}

Circular dichroism (CD) spectroscopy is commonly used to detect changes in the secondary structure of proteins ${ }^{24}$. The CD spectrum of the TTZ-PPL system was shown in Fig. 6. When the concentration ratio of PPL to TTZ was 1:0, 1:5 and $1: 10$, the $\alpha$-helix content of PPL decreased from
$10.55 \%$ to $6.48 \%$. As the concentration of TTZ increased, the absorption intensity of the negative peak gradually decreased, implying that TTZ caused a change in the secondary structure of PPL, and made the protein structure loose. But the peak shape and peak position had not been changed, indicating that the $\alpha$-helix in the PPL molecule still dominated.

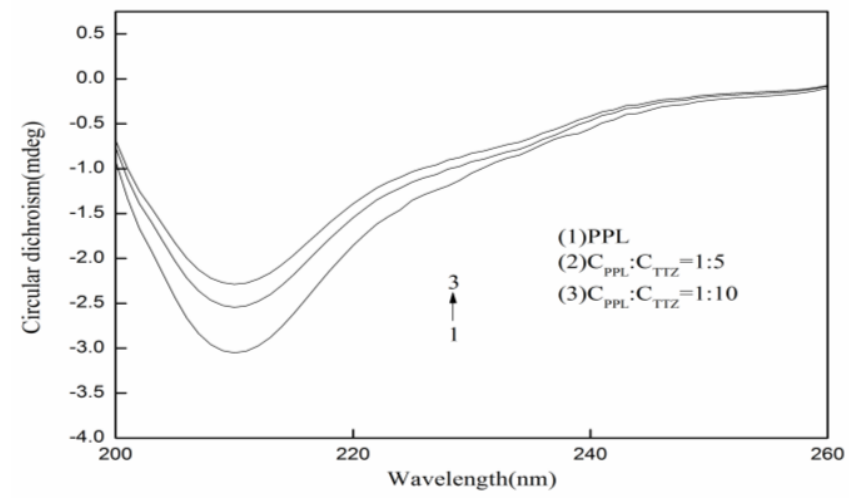

Figure 6: The circular dichroism spectra of TTZ-PPL system ( $T=298 \mathrm{~K}$ ) $C_{\mathrm{PPL}}=1.0 \times 10^{-6} \mathrm{~mol} / \mathrm{L} ; C_{\mathrm{TTZ}}=(0,1.0,2.0) \times 10^{-5} \mathrm{~mol} / \mathrm{L}$

\section{Molecular docking}

Molecular docking method can simulate the interaction between small molecules and proteins at the atomic level, so that it can find small molecules at the target protein binding site and clarify interesting biochemical processes. The best combination mode predicted by the docking software Autodock 4.2.6 was shown in Fig. 7.
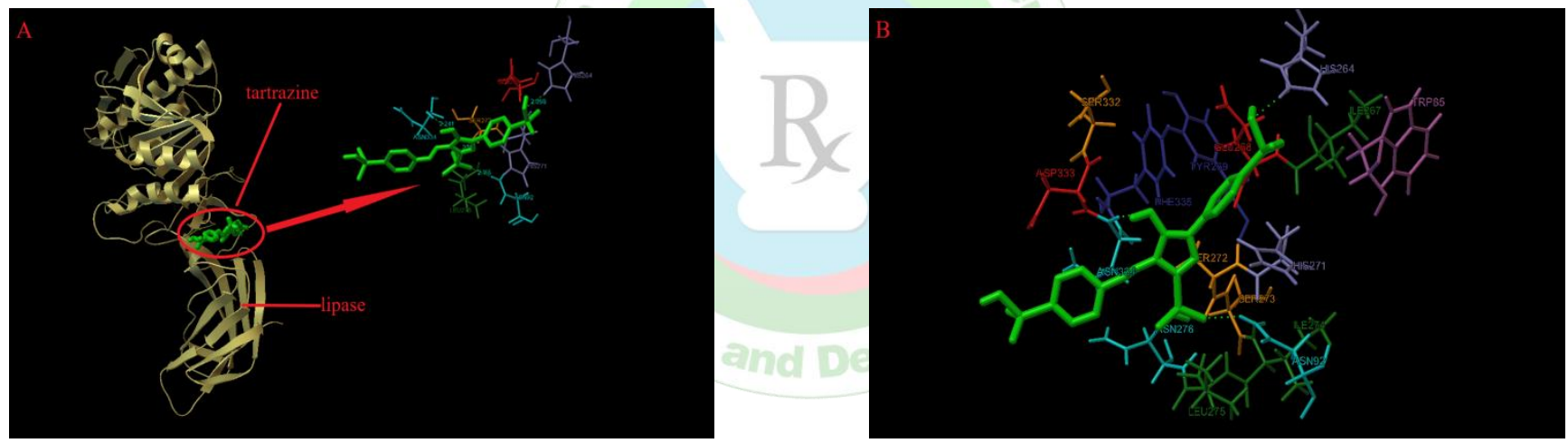

Figure 7: Computation docking model of the interaction between TTZ and PPL

TTZ located within the hydrophobic pocket in PPL (B) Detailed illustration of the amino acid residues lining the binding site in the TTZ and PPL cavity

Fig. 7 (A) showed the optimal binding configuration of the TTZ-PPL complex. The active sites of different lipases are usually composed of serine (Ser) residues and histidine (His) residues, and together with aspartic (Asp) residues or glutamic(Glu) residues to form a ternary catalytic center ${ }^{25}$. It can be seen from Fig 7(B) that the binding site of TTZ was near the active center of PPL, indicating that the binding of TTZ to PPL might affect the catalytic activity of PPL, and the binding site of TTZ was very close to the residues of Tyr269 and Trp85, as a result, the combination of TTZ and PPL could effectively quench the intrinsic fluorescence of PPL. It is consistent with the conclusion of fluorescence experiment. TTZ was surrounded by various hydrophobic residues, such as Phe335, Tyr269, Leu275, ILE274 and Trp85, which indicated that hydrophobicity promotes the binding process between TTZ and PPL. And the Asn334, Asn92, His264 residues in PPL formed three hydrogen bonds with TTZ, and the bond lengths are 2.241
$\AA$, $2.116 \AA$ and $2.098 \AA$, respectively, indicating that hydrogen bonds play a very important role in the binding of TTZ to PPL. The binding energy between TTZ and PPL obtained by molecular docking was about $-22.58 \mathrm{~kJ} / \mathrm{mol}$, which was similar to the $\Delta G$ (about- $25.33 \mathrm{~kJ} / \mathrm{mol}$ ) of TTZPPL system obtained by fluorescence experiment at $310 \mathrm{~K}$. The difference in data results may be due to the exclusion of solvents or receptors other than amino acid residues Trp,Tyr in docking simulations ${ }^{26}$. The energy data obtained by molecular docking were shown in Table 3, from which we could see that the electrostatic energy was much lower than the sum of vanderization energy, hydrogen bond energy and desolvation free energy in the process of TTZ and PPL binding. The results showed that the electrostatic interaction between TTZ and PPL was very weak, which was consistent with the effect of ionic intensity on the fluorescence experiment of TTZ-PPL binding. From the data of molecular docking, it can be further explained that 
the main force types of TTZ-PPL system were hydrophobic

interaction and hydrogen bonding interaction.

Table 3: Docking energy of TTZ-PPL system (unit: kJ/mol)

\begin{tabular}{|l|l|l|l|l|}
\hline Protein PDB ID & $\boldsymbol{\Delta} \boldsymbol{G}_{\boldsymbol{0}}$ & $\boldsymbol{\Delta} \boldsymbol{E}_{\boldsymbol{1}}$ & $\boldsymbol{\Delta} \boldsymbol{E}_{2}$ & $\boldsymbol{\Delta} \boldsymbol{E}_{3}$ \\
\hline 1OIL & -22.58 & -35.04 & -33.33 & -1.75 \\
\hline
\end{tabular}

$\Delta G_{0}$ is the binding energy in the binding process.

$\Delta E_{l}$ denotes intermolecular interaction energy, which is a sum of van der Waals energy, hydrogen bonding energy, desolvation free energy and electrostatic energy.

$\Delta E_{2}$ is the sum of van der Waals energy, hydrogen bonding energy and desolvation free energy.

$\Delta E_{3}$ is the electrostatic energy.

\section{CONCLUSION}

Under simulated physiological conditions, a variety of spectroscopy and molecular docking techniques were used to explore the interaction between TTZ and PPL. Experiments have proved that TTZ has a strong binding ability to PPL, which provides theoretical guidance for further understanding the toxicity of colorants to human and further studies are needed to provide for health risk assessment of food colorants. At present, the use of spectroscopy to study the interaction between proteins and ligands is more and more popular and favored by

\section{REFERENCES}

1. Mehedi N, Ainadtabet S, Mokrane N, Addou S, Zaoui C, Kheroua O, Saidi D. Reproductive toxicology of tartrazine (FD and C Yellow No. 5) in Swiss albino mice.[J]. American Journal of Pharmacology \& Toxicology.2009; 4(4):130-5.

2. Dorraji PS, Jalali F. Electrochemical fabrication of a novel $\mathrm{ZnO} /$ cysteic acid nanocomposite modified electrode and its application to simultaneous determination of sunset yellow and tartrazine. Food Chemistry.2017; 227:73-7.

3. Basu A, Kumar GS. Studies on the interaction of the food colorant tartrazine with double stranded deoxyribonucleic acid. Journal of Biomolecular Structure \& Dynamics.2016; 34(5):935-42.

4. McCann D, Barrett A, Cooper A, Crumpler D, Dalen L, Grimshaw K, Kitchin E, Lok K, Porteous L, Prince E, Sonuga-Brake E, Warner JO, Stevenson J. Food additives and hyperactive behaviour in 3-year-old and 8/9-year-old children in the community: a randomised, doubleblinded, placebo-controlled tria. Lancet.2007; 370(9598):1560-7.

5. Li CY, Huang ZL, He P, Wu ZQ, Chu ZZ, Wu GD, Zhan FJ. Effect of Isopropanol on Catalytic Kinetics and Molecular Spectrum of Porcine Pancreas Lipase. Chemistry \& Bioengineering.2007; 24(9):46-9.

6. Eom SH. Pancreatic Lipase Inhibitory Activity of Phlorotannins Isolated from Eisenia bicyclis. Phytotherapy Research.2013; 27(1):148-51.

7. Zhao L, Hu S, Meng Q, Hu M, Zhang H, Liu R. The binding interaction between cadmium-based, aqueous-phase quantum dots with Candida rugosa lipase. Journal of Molecular Recognition Jmr. 2018; 31(46): e2712.

8. Zhang R, Zhao L, Liu R. Deciphering the toxicity of bisphenol a to Candida rugosa lipase through spectrophotometric methods. Journal of Photochemistry \& Photobiology B Biology. 2016; 163:40-6.

9. Ma LH, Liu BS, Wang CD, Zhang HC, Cheng X. The interaction mechanism of nifedipine and pepsin[J]. Monatshefte für Chemie Chemical Monthly. 2018; 149(11): 2123-30.

10. Elmas G, Esra Y. Fluorescence interaction and determination of sulfathiazole with trypsin. Journal of Fluorescence.2014; 24(5):143945 .

11. Zhang LH, Liu BS, Li ZY, Guo Y. Comparative studies on the interaction of cefixime with bovine serum albumin by fluorescence researchers. Compared with other research methods such as chromatography, this research method has the advantages of low experimental cost and short measurement period. Therefore, this study provides an important reference for the efficient and rapid study of the interaction between proteins and ligands.

\section{ACKNOWLEDGMENTS}

The authors gratefully acknowledge the financial support of National Natural Science Foundation of China (No. 21375032).

quenching spectroscopy and synchronous fluorescence spectroscopy. Asian Journal of Chemistry. 2015; 30(5): 686-92.

12. Tayyab S, Izzudin MM,Kabir MZ, Feroz SR, Tee WV, Mahanmd SB, Alias Z. Binding of an Anticancer Drug, Axitinib to Human Serum: Fluorescence Quenching and Molecular Docking Study. Journal of Photochemistry \& Photobiology, B: Biology. 2016; 162:386-94.

13. Raza M, Yang J, Yun M, Ahand A, Khan A, Yuan QP. Insights from Spectroscopic and In-silico Techniques for the Exploitation of Biomolecular Interactions Between Human Serum Albumin and Paromomycin. Colloids and Surfaces B: Biointerfaces. 2017; 157:24253.

14. Moeinpour F, Mohseni-shahri FS, Malaekeh-nikouei B, Nassirli H. Investigation into the Interaction of Losartan with Human Serum Albumin and Glycated Human Serum Albumin by Spectroscopic and Molecular Dynamics Simulation Techniques: Acomparison Study. Chemico-Biological Interactions. 2016; 257:4-13.

15. Feroz SR, Teoh YJ, Mohamzd SB, Hong SL, Malek S, Tayyab S. Interaction of Flavokawain B with Lysozyme: A Photophysical and Molecular Simulation Study. Journal of Luminescence. 2015; 160:1019 .

16. Amroabadi MK, Taheri-kafrani A, Saremi LH, Rasteqai AA. Spectroscopic Studies of the Interaction Between Alprazolam and Apo-human Serum Transferrin as a Drug Carrier Protein. International Journal of Biological Macromolecules. 2018; 108:263-71.

17. Naik KM, Nandibewoor ST. Spectroscopic studies on the interaction between chalcone and bovine serum albumin. Journal of Luminescence. 2013; 143:484-91.

18. Salam M, Rokonujjaman M, Rahman A, Sultana UN, Sultan N. Study of in Vitro Interaction of Sildenafil Citrate with Bovine Serum Albumin by Fluorescence Spectroscopy [J]. Pharmacology \& Pharmacy. 2015; 6(2):94-101.

19. Ross PD, Subramanian S. Thermodynamics of protein association reactions: forces contributing to stability. Biochemistry. 1981; 20(11):3096-102

20. Jahanban-Esfahlan A, Panahi-Azar V, Sajedi S. Spectroscopic and molecular docking studies on the interaction between N-Acetyl Cysteine (NAC) and bovine serum albumin[J]. Biopolymers. 2015; 103(11): 638-45.

21. Buddanavar AT, Nandibewoor ST. Multi-spectroscopic characterization of bovine serum albumin upon interaction with 
atomoxetine. Journal of Pharmaceutical Analysis. 2017; 7(3):148-55.

22. Hu X, Yu Z, Liu R. Spectroscopic investigations on the interactions between isopropanol and trypsin at molecular level. Spectrochim Acta A Mol Biomol Spectrosc. 2013; 108:50-4.

23. Cagnardi P, Villa R, Gallo M, Locatelli C, Carli S, Moroni P, Zonca A. Cefoperazone sodium preparation behavior after intramammary administration in healthy and infected cows. J. Dairy Sci. 2010; 93(9): 4105-10.

24. Bhogale A, Patel N, Mariam J, Donger PM, Miotello A, Kothari DC. Comprehensive studies on the interaction of copper nanoparticles with bovine serum albumin using various spectroscopies. Colloids Surf B Biointerfaces. 2014; 113(13):276-84.

25. Fan ZF, Zeng WC, Dai JL, He Q. Interaction of Epigallocatechin-3gallate with Porcine Pancreas Lipase. Food Science. 2013; 34(7):20-3.

26. Jana S, Dalapati S, Ghosh S, Guchhait N. Study of microheterogeneous environment of protein Human Serum Albumin by an extrinsic fluorescent reporter: a spectroscopic study in combination with Molecular Docking and Molecular Dynamics Simulation. Journal of Photochemistry \& Photobiology B Biology. 2012; 112(231):48-8. 